\title{
Mobility based 3D simulation of selective, viscoelastic polymer reflow using SURFACE EVOLVER
}

\author{
Robert Kirchner ${ }^{\text {a) }}$ and Helmut Schift ${ }^{\text {b) }}$ \\ Laboratory for Micro- and Nanotechnology, Paul Scherrer Institut, 5232 Villigen PSI, Switzerland
}

(Received 27 June 2014; accepted 15 September 2014; published 2 October 2014)

\begin{abstract}
This work demonstrates implementation of the main effects of viscoelastic thermal polymer reflow in an efficient energy and mobility based simulation. The concept is based on a finite-element, soap-film method using the free software surface Evolver. Properties of a homogeneous 3D volume are thereby represented by a corresponding $2 \mathrm{D}$ surface. The simulation only requires the contact angle between polymer and substrate for infinite long reflow times, obtained from fingerprint experiments, and a mobility value as input. The mobility value is a measure for the polymer-chain mobility and is directly linked to the polymer viscosity. This concept allows for an accurate and fast treatment of the thermomechanically complex polymer behavior close to the glass transition. The simulation time scale is linearly related to the experimental time scale allowing for accelerated-time simulations. Simulation and experiment showed a very good agreement. As a generalized concept, the approach presented here can be used for fast and full 3D shape computation during any complex, energy driven geometry optimization process like polymer reflow, viscoelastic wetting or dewetting and droplet coagulation. This simulation may facilitate a faster uptake of grayscale reflow technologies for industrial processes. Supplementary material supports a quick grasp of the simulation approach. (C) 2014 American Vacuum Society.
\end{abstract}

[http://dx.doi.org/10.1116/1.4896480]

\section{INTRODUCTION}

Three-dimensional manufacturing is crucial for future devices in a broad size range for the fields of fluidics and photonics. Polymer materials are promising for these areas of application due to a broad spectrum of available polymers and emerging patterning technologies ${ }^{1,2}$ as well as the potential for efficient tuning of material properties. ${ }^{3,4}$ Reflowing lithographically prepatterned, binary polymer structures is an established method for microlens fabrication. 5,6 Grayscale electron beam lithography (GEBL) has demonstrated its potential for multitier polymer structures down to the $10 \mathrm{~nm}$ regime. ${ }^{7}$ GEBL was also used as a direct writing lithography method for the fabrication of curved surfaces. ${ }^{8}$ A combined GEBL and thermal reflow approach recently brought enhanced structural variety ${ }^{9}$ to the lithography portfolio for optics and fluidics devices. This combined method is based on selective reflow of the GEBL exposed structures to give ultrasmooth surfaces. The process is referred to as temperature activated selective topography equilibration (TASTE). ${ }^{10}$ During the TASTE reflow, the evolution of the geometry over time is governed by locally varied polymerchain mobility and is driven by energetic imbalances toward an energy optimal shape. ${ }^{11}$

The ability to simulate and forecast energy-optimizing behavior of polymers is essential for quick realization of new design ideas and products using processes like TASTE. SURFACE EVOLVER (SE) soap-film modeling was introduced for $3 \mathrm{D}$ polymer shape prediction, and the transition of rectangular polymer patterns into ridges with a rounded shape

\footnotetext{
${ }^{a}$ Electronic mail: robert.kirchner@psi.ch

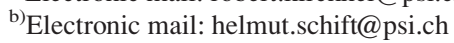

was analyzed. ${ }^{11}$ This can be applied to lens fabrication starting from a resist pattern with a single, homogeneous viscosity. The existing method is now extended to structures where a range of viscosities are present. To simulate such inhomogeneous structures, a new simulation principle using different mobility values within a finite element (FE) mesh is introduced. To demonstrate the new principle, exemplary double-step structures, i.e., asymmetric structures consisting of two different viscosities, were used. By showing the ability to simulate and forecast the full 3D thermal reflow behavior of such basic geometries, the way is paved to extent existing energy and mobility based soapfilm modeling toward grayscale lithography and the prediction of much more complex geometries. The structures that can now be simulated contain, for example, multiple sections of different viscosities resulting in a different reflow behavior. This will enable a quick turnaround for design and testing of structures within a vast range of applications.

\section{EXPERIMENT}

\section{A. Dose modulated EBL}

Dose modulated electron beam grayscale lithography is used to write double-step patterns into poly(methyl methacrylate) (PMMA) [Fig. 1(a)]. The resist was mr-I PMMA120k (micro resist technology $\mathrm{GmbH}$ ). The double-steps were realized by applying a three-dose-grayscale lithography. One dose is the dose-to-clear $D_{c}$. It was applied to obtain freestanding polymer lines. The remaining doses define the height of the two individual steps within these lines. An unexposed section $\left(D_{0}=0\right)$ was used to give the original resist height and an exposed section $\left(D_{c}>D_{x}>D_{0}\right)$ gave a reduced height. Due to the locally varied electron beam dose 


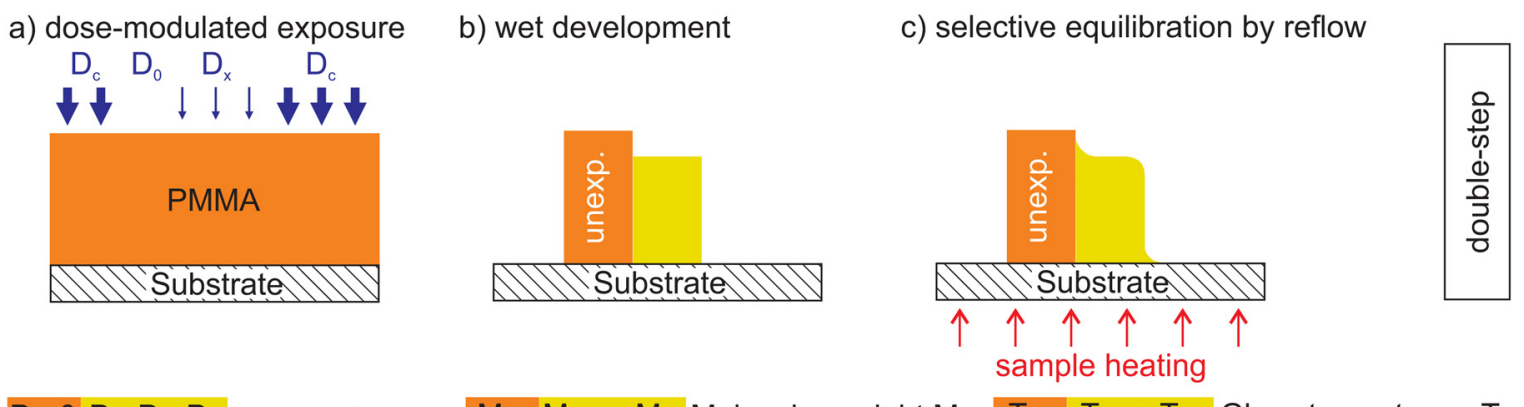

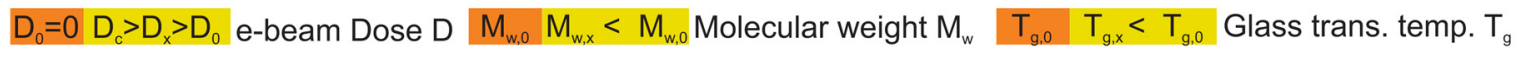

FIG. 1. (Color online) Process scheme used to obtain reflowed double-step structures by (a) electron beam grayscale exposure, (b) solvent based development, and (c) global reflow on a hotplate.

(100kV, EBPG 5000 Plus, VISTEC), the PMMA molecular weight $^{10,12}$ and consequently the development rate in 4methylpentan-2-one (methyl isobutyl ketone) varied across the pattern. As a result, different step heights were obtained after development [Fig. 1(b)]. Additionally, the differing molecular weight directly influences the glass transition temperature $T_{g}$ and the reflow behavior. ${ }^{10}$ Thus, the resulting double-step shape was defined by $D_{x}$ determining the etch rate, the glass transition temperature, and consequently the reflow behavior. Within a step, these properties are assumed to be homogeneous. About $100 \mu \mathrm{m}$, long lines with cross sections as depicted in Fig. 2 were realized as initial structures and were subsequently reflowed [Fig. 1(c)]. Similar to previous work, ${ }^{11}$ cross sections of the reflowed structure (Fig. 3) were obtained, after manual, crystal oriented cleaving of the respective structures on the silicon substrates, by a scanning electron microscope (SEM).

\section{B. Reflow}

Thermoplastic reflow can be understood as a viscoelastic creep process under a continuous force. The force originates from the differences in surface tension and the surface free energy of a thermoplastic resist and the substrate it is placed on, respectively (Fig. 4). ${ }^{11}$ The difference in $T_{g}$ leads to a different reflow behavior of the stepped structures ${ }^{11}$ under the same continuous force. The lower the molecular weight, the faster the reflow proceeds to the final optimal shape due

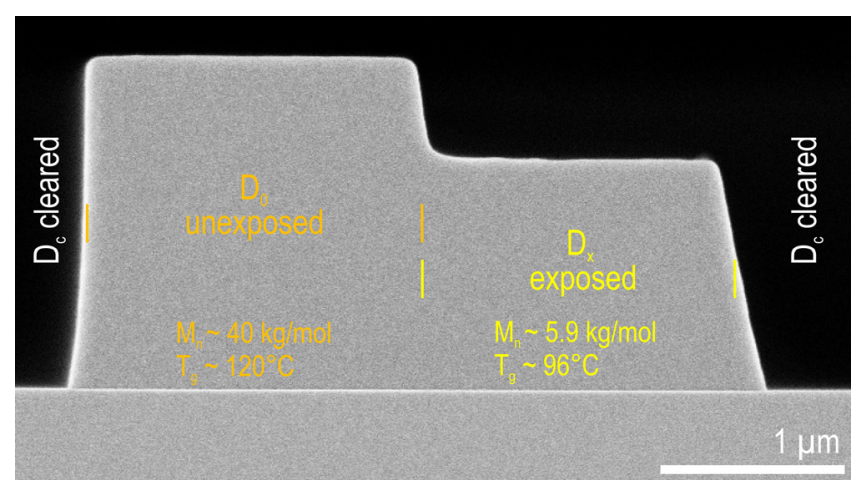

FIG. 2. (Color online) SEM cross section of a PMMA double-step structure on a silicon substrate after grayscale electron beam writing and wet development $\left[M_{w}\right.$ (Ref. 10) and $T_{g}$ (Ref. 10)]. to the lower viscosity, which is equal to a higher polymer chain mobility.

The double-step geometry (cf. Fig. 2) was forced by the electron grayscale patterning and wet development. It is energetically not very favorable. It comprises sharp corners and an angle between resist and substrate of about $90^{\circ}$. If the polymer chains are given energy to move, this geometry will undergo reflow to obtain an energetically more favorable shape (cf. Fig. 3). This means a geometry with more rounded, and thus less high frequency structural contributions, and a geometry with a smaller apparent contact angle $\alpha$. This angle depends on the molecular weight and the reflow time. The molecular weight dependent, asymptotic, apparent contact angle obtained for very long reflow times ${ }^{11}$ was used as approximation for the contact angle that would be found for an infinite reflow time, which is required in the simulation model.

\section{SOAPFILM MODELING}

An energy based approach was applied to model the reflow behavior using the free software SE. ${ }^{11,13} \mathrm{SE}$ is based on a FE simulation core. A 3D geometry is implemented as collection of 2D FE surfaces representing only the geometry's "outer
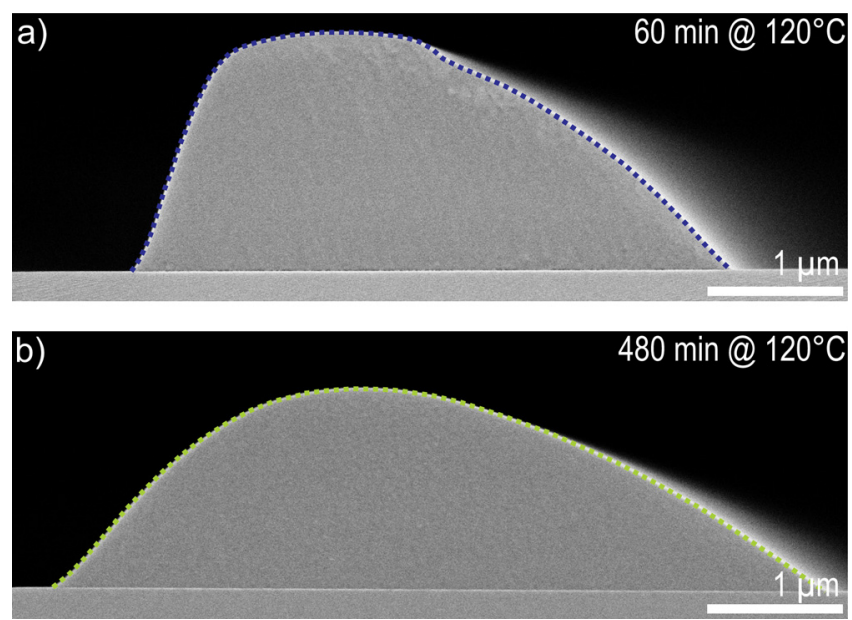

FIG. 3. (Color online) Exemplary SEM cross sections of PMMA double-step structures on a silicon substrate, reflowed at $120^{\circ} \mathrm{C}$ for (a) $60 \mathrm{~min}$ and for (b) $480 \mathrm{~min}$. Only the dotted contour is required for further experimental analysis (e.g., cf. Fig. 8) and comparison with the simulation (e.g., cf. Fig. 13). 


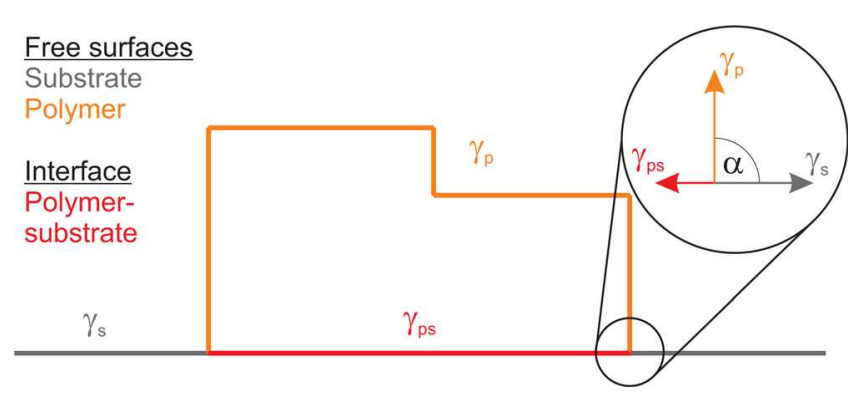

FIG. 4. (Color online) Schematic cross section and energetic situation of a double-step thermoplastic resist structure after wet development and before undergoing thermal reflow. The zoomed-in section shows the angle $\alpha$ as a results of the lithography process. During reflow this angle becomes an (apparent) contact angle defined by the respective surface and interface energies $\gamma$.

shell." This is termed soapfilm modeling and means the structure interior does not contain finite elements (Fig. 5). During simulation, the volume is kept constant. For such a simulation, the structural properties of the simulated 3D entity must be represented by properties of the $2 \mathrm{D}$ surfaces. In this work, triangulated 2D surfaces were used. One single finite surface element (triangle) is termed a facet in SE and is defined by three nodes, termed vertices in $\mathrm{SE}$, of the FE mesh. The vertices are connected by oriented edges (Fig. 6). SE allows a huge spectrum of possible energies to be assigned like gravitational energy, surface energy, and further different implementations of mean and Gaussian curvature. ${ }^{14}$ In this work, only surface energy was required. Each facet is assigned a certain surface energy. In case of the solid polymer surface, it is the surface tension of the polymer at the respective reflow temperature. For the experimentally applied PMMA, a fixed value of $33.5 \mathrm{mN} / \mathrm{m}$ (Ref. 15) was used for both the 110 and $120^{\circ} \mathrm{C}$ reflow. The double-step structure is simulated as being a line of infinite length. Practically, this is implemented by periodic boundary conditions (mirror planes) at the limiting ends of a $5 \mu \mathrm{m}$ long simulated region. Therefore, the contact angle with these mirror planes was fixed to $90^{\circ}$ to represent a periodic boundary condition. The contact angle to the substrate was implemented via the interface energy between polymer and substrate. ${ }^{11}$ This contact angle was obtained from long-term reflow experiments and defines the minimal energy state SE tries to converge to.

In the SE simulation, the total energy of the system $E_{\mathrm{tot}}$ is the surface energy of the free polymer surface $E_{p}$ minus the energy stored in the interfaces between polymer and substrate

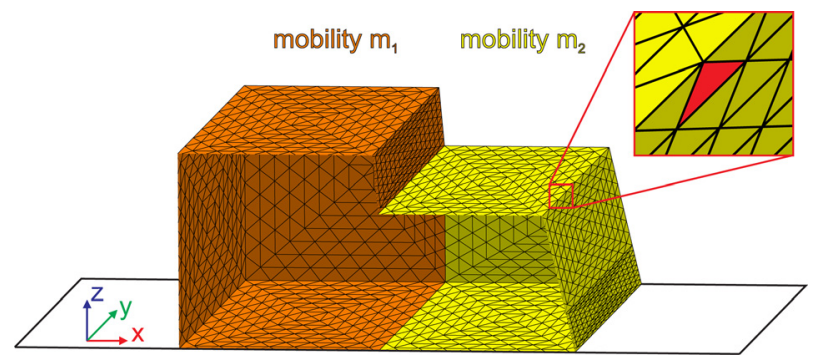

FIG. 5. (Color online) Cross section of the triangulated FE model of the double-step in Fig. 2 showing the FE surface (zoom in) and the specialty of a soapfilm model (structure interior not meshed).

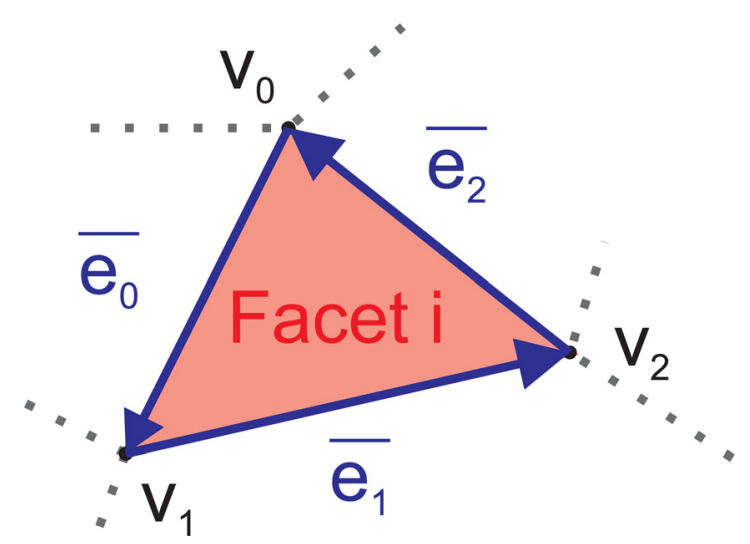

FIG. 6. (Color online) Schematic, triangular FE facet $i$ used for surface meshing consisting of vertices $v$ and oriented edges $\bar{e}$.

$E_{p s}$ and in the mirror planes $E_{m}$ [Eq. (1)]. The physical interpretation is that the geometry reduces the total system energy by decreasing the free polymer area and increasing the interface areas. This is physically consistent with real wetting behavior

$$
E_{\mathrm{tot}}=E_{p}-\left(E_{p s}+E_{m}\right) .
$$

The respective energies $E_{x}$ are calculated as sum over the energies $E_{x, i}$ of all $n$ individual facets $i$ within the three different regions: free polymer surface $(x=p)$, polymer-substrate interface $(x=p s)$, and mirror planes $(x=m)$ [Eq. (2)]. The individual facet energies $E_{x, i}$ are calculated from the surface tension or interface energy $\gamma$ of a facet $i$ and the area of this single facet [Eq. (3)]. This is why the contact angle must be implemented via a surface or interface energy

$$
\begin{aligned}
& E_{x}=\sum\left[E_{x, i}\right]_{0}^{n} \mid \text { with }(x=p, p s, m), \\
& E_{x, i}=\frac{\gamma_{i}}{2}\left|\overline{e_{0}} \times \overline{e_{1}}\right| .
\end{aligned}
$$

The force on each vertex is calculated from the gradient of the energy field determined above and the position of the vertex within that energy field. ${ }^{14} \mathrm{SE}$ was operated in the area normalization mode to approximate a vertex motion by mean curvature, i.e., a surface tension flow. In this mode, the velocity of a vertex is indirectly proportional to the area of the facets surrounding this vertex. As each facet $i$ has three vertices associated with it, the relative area contribution to the force of one vertex is $1 / 3$ the area of the surrounding facets $A$. The vertex force $\bar{F}$ gives the velocity $\bar{\nu}$ of movement of this vertex in the area normalization mode as

$$
\bar{\nu}=\frac{3}{A} \bar{F} \cdot m .
$$

The important point is SE allows for the definition of a local mobility value $m$ for each vertex. The inverse of this simulation parameter is a resistance to motion and enables implementation of differences in viscosity, which is also a measure for the resistance to motion. SE uses a so-called global scale factor $s$ with which the velocity $\bar{\nu}$ is multiplied to give the vector $\bar{\delta}$ of movement [Eq. (5)]. The scale factor is in this case a physical representation of time. The continuous sum of 


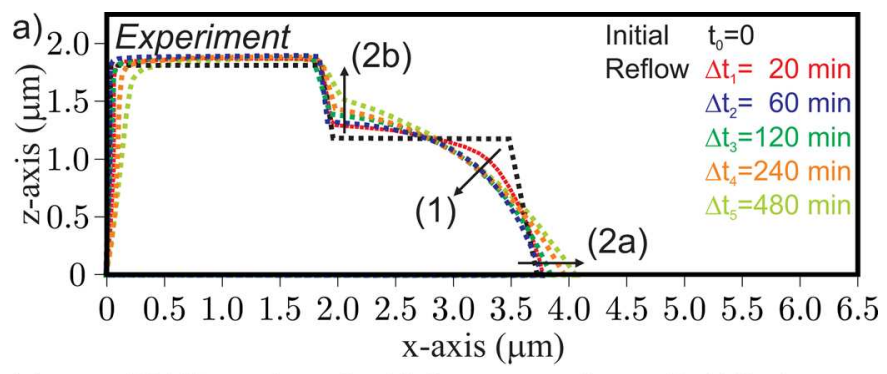

b)

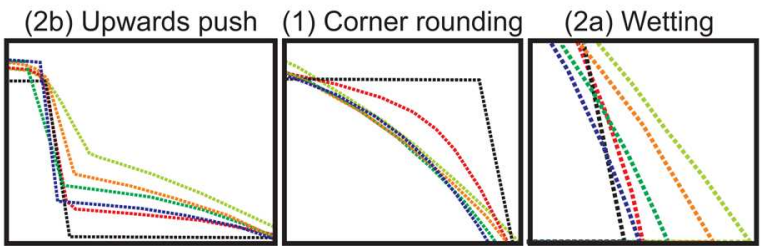

FIG. 7. (Color online) (a) Experimental contours of scanning electron microscopy cross sections of a double-step structure (cf. Fig. 3 for principle) after reflow at $110^{\circ} \mathrm{C}$ for different durations of time and (b) additional zoomed in sections showing details of the (1) corner rounding (inwards pull) as well as the simultaneous (2a) wetting and (2b) upwards push of the polymer.

the scale factor used in each iteration is the total time of the simulated process. SE was operated in a way that allows the SE kernel to optimize the scale factor itself for a fast and smooth convergence toward the final, energy optimal shape

$$
\bar{\delta}=\bar{\nu} \cdot s .
$$

Basically, SE tries to minimize the total energy by moving the vertices with an optimized $\bar{\delta}$ and by following the total system energy gradient. In contrast to the simulation approach published before, ${ }^{11}$ only the contact angle for infinite reflow time was used instead of implementing a relationship for the contact angle over time. The final contact angle of the exposed step was set to $55^{\circ}$ and $25^{\circ}$ for the reflow at 110 and $120^{\circ} \mathrm{C}$, respectively. The corresponding contact angles for the unexposed step were $80^{\circ}\left(110^{\circ} \mathrm{C}\right)$ and $55^{\circ}\left(120^{\circ} \mathrm{C}\right)$.

\section{RESULTS AND DISCUSSION}

\section{A. Experimental reflow observations}

It was clearly visible that the exposed step, in general, reflowed faster than the unexposed step. Figure 7 depicts the time sequence of a reflow at $110^{\circ} \mathrm{C}$ for $20-480 \mathrm{~min}$. The reflow temperature was below the $T_{g}$ of the unexposed step $\left(120^{\circ} \mathrm{C}\right)$, while it was above the $T_{g}$ of the exposed step $\left(96^{\circ} \mathrm{C}\right.$ ) (cf. Fig. 2). ${ }^{10}$ As discussed elsewhere, ${ }^{11}$ the rounding of the free polymer corners [Fig. 7(1)] proceeds faster compared to the wetting of the polymer-substrate contact line [Fig. 7(2a)]. Together with the fact that the chain mobility within the unexposed step is quite low, this leads to material being pushed upwards [Fig. 7(2b)] during the reflow to accommodate the corner rounding while keeping the volume constant. Wetting and upwards push proceeded with approximately the same speed. This can be explained by the fact that the mobility of the exposed step, where the majority of those effects take place, is homogeneously distributed. The corner rounding of the unexposed step is much smaller due to the much higher viscosity and thus the slower movement.
Figure 8 depicts the same time sequence for the reflow at $120^{\circ} \mathrm{C}$. In comparison to Fig. 7 , it can be seen that the difference in viscosity between the unexposed and the exposed step is smaller for the $120^{\circ} \mathrm{C}$ (cf. Fig. 8) than for the $110^{\circ} \mathrm{C}$ reflow (cf. Fig. 7). This is demonstrated by the more similar slopes of the unexposed and the exposed step for the higher temperature. Within the same time steps, the footing of the exposed step moves much further for the higher reflow temperature (improved wetting). This is due to the lower viscosity of the exposed material at the higher temperature. Furthermore, the apparent polymer-substrate contact angle for the $480 \mathrm{~min}$ reflow was smaller for the higher temperature. This is in good agreement with earlier observations. ${ }^{11}$

\section{B. Energy and mobility based simulation}

See the supplementary material for the simulation script and a real-time video showing the course of the $3 \mathrm{D}$ reflow at $120^{\circ} \mathrm{C}$. Such a simulation required about $3 \mathrm{MB}$ total data memory and took less then 2 min on a 64 bit Intel i5 $1.8 \mathrm{GHz}$ dual-core equipped notebook with $8 \mathrm{MB}$ RAM. The supplementary material contains the geometry file defining the geometry parameters, the simulation file containing the initialization and parametrization of the simulation space as well as a video ${ }^{16}$ showing a real-time $3 \mathrm{D}$ simulation of the reflow at $120^{\circ} \mathrm{C}$ (cf. Fig. 13).

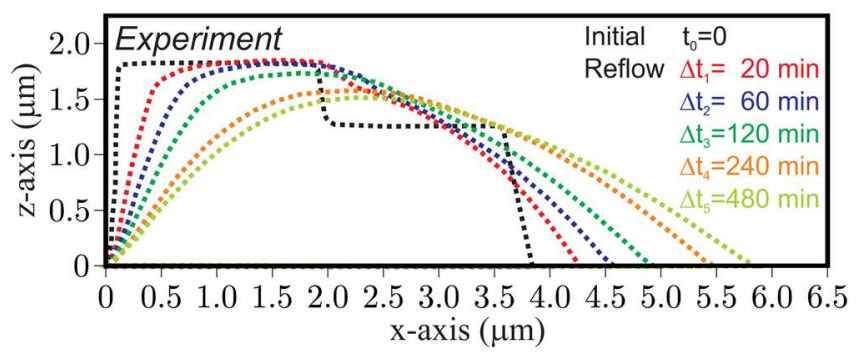

FIG. 8. (Color online) Experimental contours of scanning electron microscopy cross sections of a double-step structure after reflow at $120^{\circ} \mathrm{C}$ for different durations of time (cf. Fig. 2). 


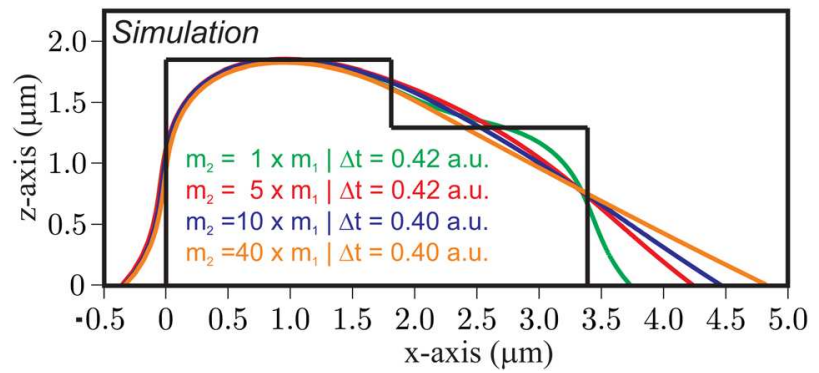

FIG. 9. (Color online) Reflow simulation of a double-step at the same point of time with different mobility values and the same contact angle for infinite reflow time.

\section{Effect of the virtual mobility value}

A principle proof for the physical correctness of the simulation, using different mobilities $m$ for the unexposed $\left(m_{1}\right)$ and the exposed step $\left(m_{2}\right)$, is depicted in Fig. 9. The mobility ratio $m_{2} / m_{1}$ was increased from 1 up to 40 . The higher the relative mobility of the exposed step was set, the faster it moved. Additionally, higher mobilities led also to more pronounced corner rounding in the same time. Due to this faster reflow, the step structure was transformed more into a wedge like structure for the higher value of $m_{2}$. This behavior agrees well with the experimental observations (cf. Sec. IV A), indicating that the simulation is physically correct. The low $m_{2} / m_{1}$-ratio resembles more the reflow at $110^{\circ} \mathrm{C}$, or for a short time, while the large $m_{2} / m_{1}$-ratio represents more the $120^{\circ} \mathrm{C}$, or long time reflow. This suggests the applicability of a time-temperature superposition. However, as shown in Ref. 11, different reflow temperatures have different contact angles for infinite reflow times. The superposition is only useable for low molecular weight compounds with a comparable contact angle. For large difference in the contact angle, as for the reflow at $110^{\circ} \mathrm{C}$ compared to $120^{\circ} \mathrm{C}$, this superposition is not applicable. Simulations showed a much better agreement with the experiment when the correct contact angle after infinite reflow time at a certain reflow temperature was used instead of using the smallest observed contact angle only (data not presented).

As the mobility of the unexposed step remains constant, the distance its footing moves as well as the shape of the unexposed step also is identical for the same simulation time. However, a slight reduction in height of the unexposed step can be observed in the simulation for very high $m_{2}$-values. This is a physically realistic behavior. Due to a material pull by the right hand, reflowing part, a material displacement to the right is the consequence.

\section{Simulation based mobility extraction}

The mobility ratio was empirically optimized to obtain the best data fit between the experimental and simulated cross sections. Therefore, first the longest reflow time was used. Once a good fit was obtained for the longest reflow time, the other simulation results were obtained by correlating the accumulated scale factors for each iteration step, representing the simulation time (total time), with the real experimental time. Thus, the total time for the shape profile best fitting to

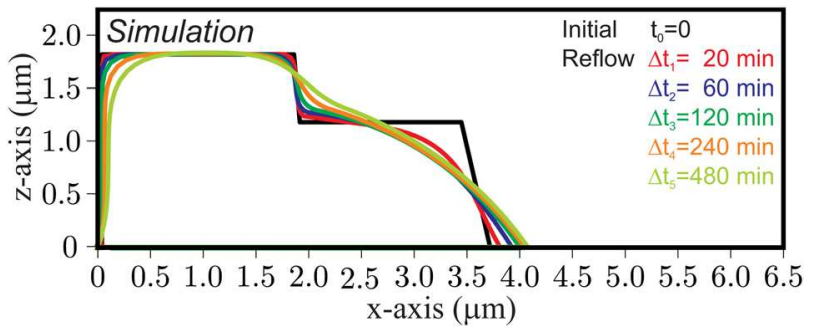

FIg. 10. (Color online) Reflow simulation of a double-step at $110^{\circ} \mathrm{C}$ using a contact angle of $55^{\circ}$ (exposed step) and a $m_{2} / m_{1}$-ratio of 50 .

the 480 min reflow was multiplied by 0.5 to get the profile for $240 \mathrm{~min}$. This was repeated until an optimal agreement was found for all reflow times. It is important to note that this linear relationship between the real reflow time and the simulation time was always applied. The simulated shape evolution for the optimal $m_{2} / m_{1}$-ratio of a two-step structure at $110^{\circ} \mathrm{C}$ is depicted in Fig. 10 and for a $120^{\circ} \mathrm{C}$ reflow in Fig. 11. Very similar to Fig. 7, a fast corner rounding with a relative slow wetting can be observed in the simulation at $110^{\circ} \mathrm{C}$. Unlike the experiment, no movement of the unexposed material upwards is observed in the simulation. As in Fig. 8, a faster reflow is observed for the higher reflow temperature Fig. 11.

\section{Comparison of reflow experiment and simulation}

The detailed comparison for each time step is summarized in Fig. $12\left(110^{\circ} \mathrm{C}\right)$ and Fig. $13\left(120^{\circ} \mathrm{C}\right)$. First of all, there was a very good qualitative agreement between the simulation and the experiment for all steps in the time-sequence and for both temperatures. Second, there was also a quantitatively high agreement between simulation and experiment. The empirically optimized $m_{2} / m_{1}$-ratio was 50 and 5 for the $110^{\circ} \mathrm{C}$ and $120^{\circ} \mathrm{C}$ reflow, respectively. This is consistent with experimental observations: for the higher reflow temperature, the difference in viscosity is lower. The comparison of simulation and experiment for an additional geometry, having a smaller exposed step, showed the same good agreement (Fig. 14). This demonstrates the broad applicability of the presented approach. The optimal data fitting was obtained for a slightly reduced $m_{2} / m_{1}$-ratio of 2 . Thus, the mobility of the exposed step is also influenced by the unexposed step.

A difference between experiment and simulation can be found especially for the lefthand wall and corner of the unexposed step as well as in the transition region between the two steps. Considering the forward scattering of the electron

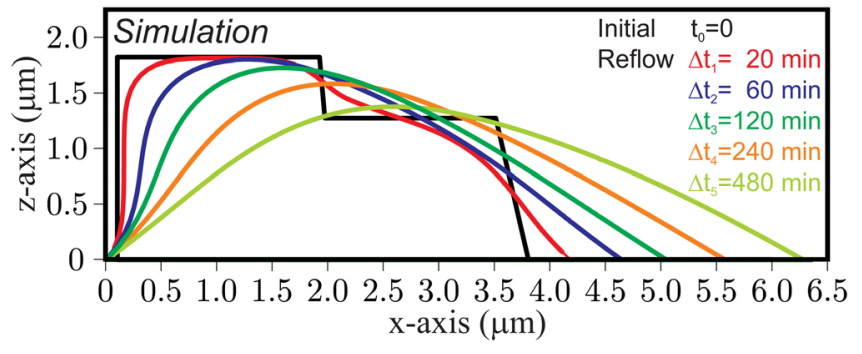

Fig. 11. (Color online) Reflow simulation of a double-step at $120^{\circ} \mathrm{C}$ using a contact angle of $25^{\circ}$ (exposed step) and a $m_{2} / m_{1}$-ratio of 5 . 

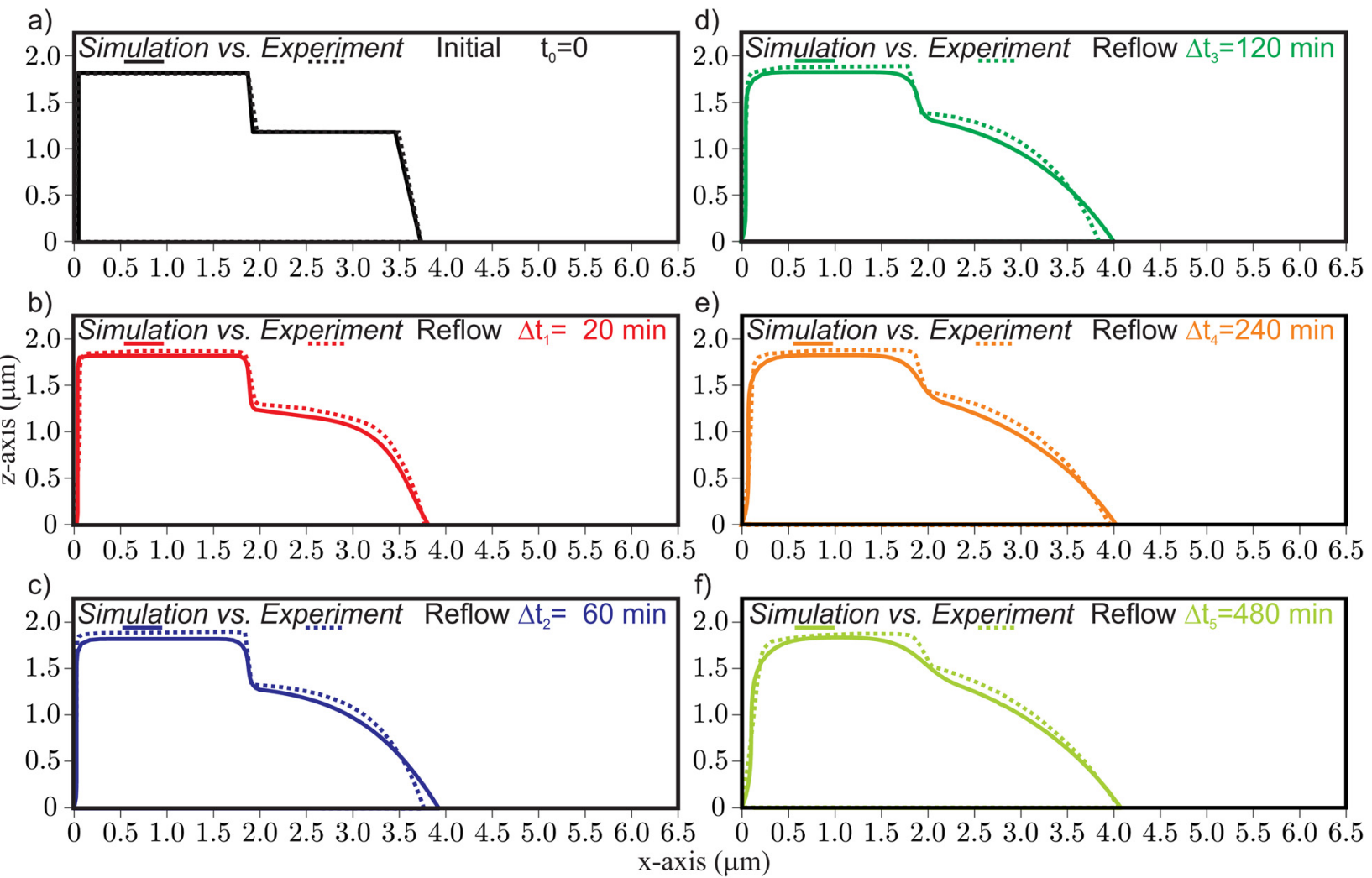

FIg. 12. (Color online) Comparison of simulated profiles and experimental SEM cross section contours for reflow at $110^{\circ} \mathrm{C}$ and reflow times from (a) 20 min up to (f) $480 \mathrm{~min}$. The data were obtained for $m_{2} / m_{1}=50$.
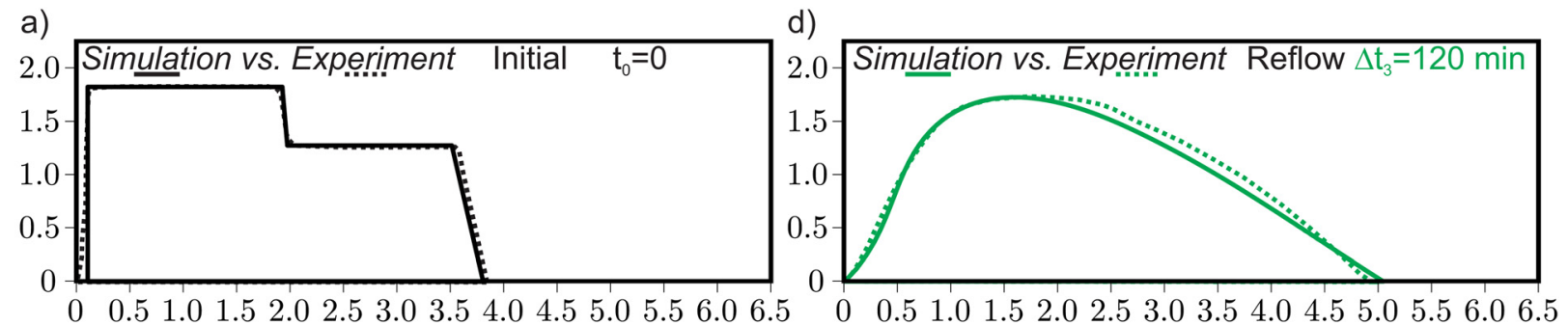

b)

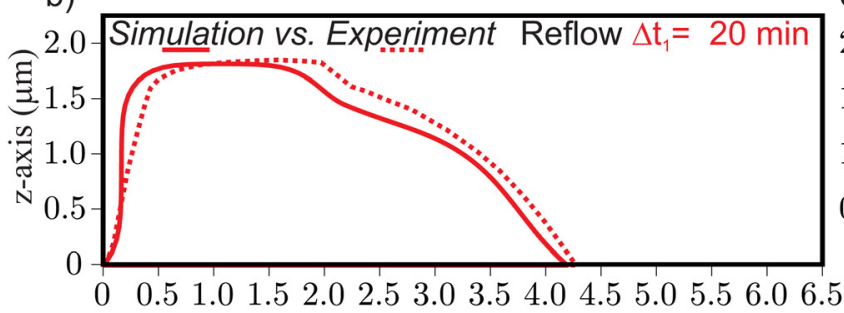

e)

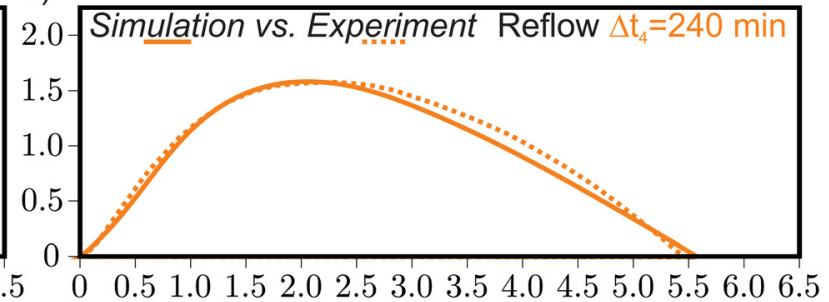

c)

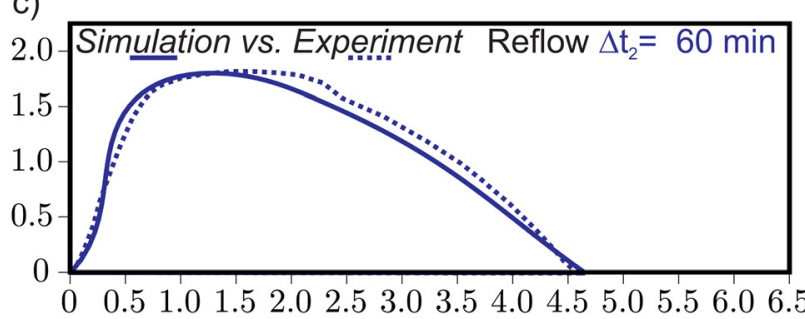

f)

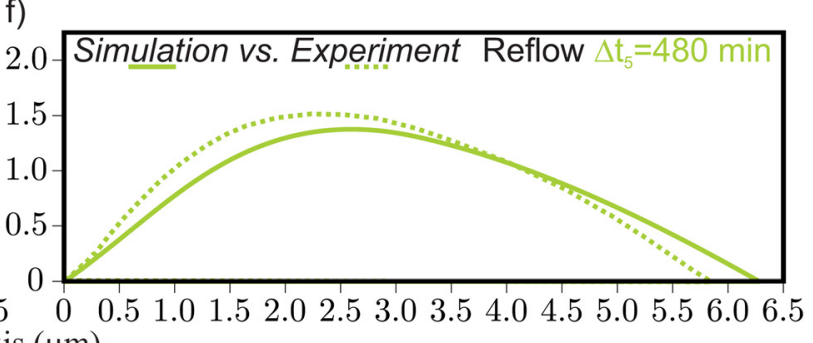

FIG. 13. (Color online) Comparison of simulated profiles and experimental SEM cross section contours for reflow at $120^{\circ} \mathrm{C}$ and reflow times from (a) 20 min up to (f) $480 \mathrm{~min}$. The data were obtained for $m_{2} / m_{1}=5$. 


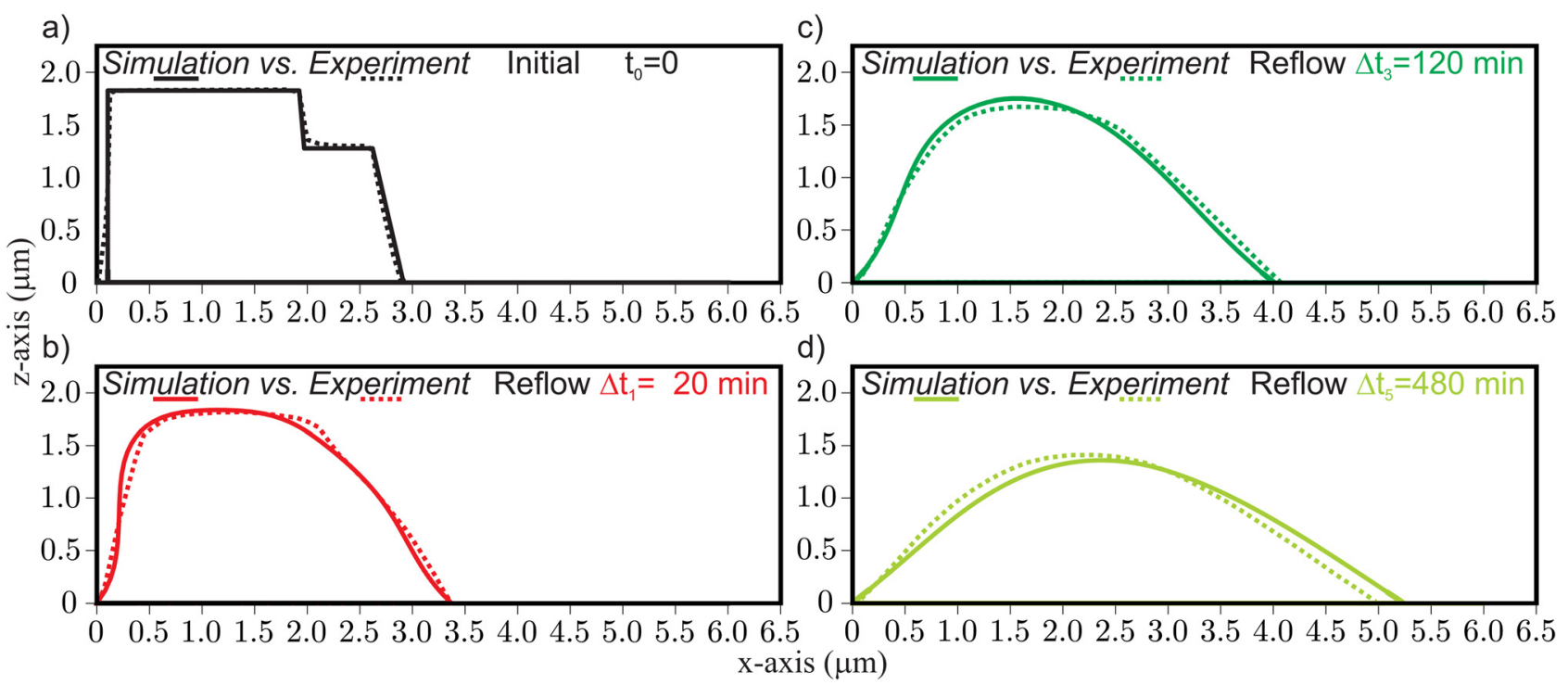

FIG. 14. (Color online) Comparison of simulated profiles and experimental SEM cross section contours for reflow at $120^{\circ} \mathrm{C}$ and reflow times from (a) 20 min up to (d) $480 \mathrm{~min}$. The data were obtained for $m_{2} / m_{1}=2$.

beam and the fact that an abrupt transition of the molecular weight, and thus the $T_{g}$, at the structure edge as well as in the transition region between the steps is not possible, it becomes clear that both sections need a special treatment. This is a true volume behavior and can only be partly considered in the soapfilm model. For the transition region between the two steps, an average mobility was assumed. However, a reduced mobility at the structures lateral periphery, due to the dose-to-clear, was not implemented.

The good agreement with the experiment first of all shows that the implemented model successfully follows the real behavior of the reflow even though only the contact angle for infinite reflow time and a mobility value was implemented. Easily interpreted, SE follows iteratively the same principle as the reflow: surface free energy differences create a force which drives the geometry toward minimal energy shapes. The speed of this movement depends mainly on the molecular mobility, i.e., the viscosity. The surface, both in the experiment and in the simulation, moves step-by-step toward the energy optimal shape. Second, this agreement shows that the simulation time is linearly related to the real experimental time scale. All intermediate simulation shapes represent real solutions for a well-defined reflow time. This allows for accelerated-time simulations.

\section{CONCLUSIONS}

The SURFACE EVOLVER is an efficient tool to simulate and forecast the viscoelastic thermal reflow behavior of complex geometries in full 3D. The energy based soapfilm modeling of thermal reflow achieves a very high agreement with experimental results. The parametrization of the simulation is done only by two values which are the contact angle for infinite reflow time and a mobility ratio. These major parameters were identified as being sufficient to accurately describe the reflow behavior. Based on the few input parameters, the agreement with the experiment in a thermomechanically very complex region like the glass transition region is extraordinary. Together with the fast computation of the soapfilm model, this approach is very interesting for industrial uptake of reflow modeling. Future work will focus on the relationship between the real polymer viscosities, the electron beam doses, and the mobility values.

\section{ACKNOWLEDGMENTS}

The authors would like to express there special thanks to V. A. Guzenko for the helpful discussions as well as C. Padeste and K. Vogelsang for the support during the course of this work. Additional thanks go to K. A. Brakke for the extremely valuable support and discussion at several points of this work. Finally, the authors especially thank micro resist technology $\mathrm{GmbH}$ for providing the PMMA resist.

${ }^{1}$ H. Ma, A. K.-Y. Jen, and L. R. Dalton, Adv. Mater. 14, 1339 (2002).

${ }^{2}$ L. J. Guo, Adv. Mater. 19, 495 (2007).

${ }^{3}$ Y. Ofir, I. W. Moran, C. Subramani, K. R. Carter, and V. M. Rotello, Adv. Mater. 22, 3608 (2010).

${ }^{4}$ R. Kirchner, M.-K. Kaiser, B. Adolphi, R. Landgraf, and W.-J. Fischer, Phys. Status Solidi A 208, 1308 (2011).

${ }^{5}$ Y. Ishihara and K. Tanigaki, Int. Electron Devices Meet. 29, 497 (1983).

${ }^{6}$ Z. D. Popovic, R. A. Sprague, and G. A. N. Connell, Appl. Opt. 27, 1281 (1988).

${ }^{7}$ F. Hu and S.-Y. Lee, J. Vac. Sci. Technol., B 21, 2672 (2003).

${ }^{8}$ Y. Hirai, S. Harada, H. Kikuta, Y. Tanaka, M. Okano, S. Isaka, and M. Kobayasi, J. Vac. Sci. Technol., B 20, 2867 (2002).

${ }^{9}$ A. Schleunitz and H. Schift, J. Micromech. Microeng. 20, 095002 (2010).

${ }^{10}$ A. Schleunitz, V. Guzenko, M. Messerschmidt, H. Atasoy, R. Kirchner, and H. Schift, Nano Converg. 1, 1 (2014).

${ }^{11}$ R. Kirchner, A. Schleunitz, and H. Schift, J. Micromech. Microeng. 24, 055010 (2014).

${ }^{12}$ E. A. Dobisz, S. L. Brandow, R. Bass, and J. Mitterender, J. Vac. Sci. Technol., B 18, 107 (2000).

${ }^{13}$ K. A. Brakke, Exp. Math. 1, 141 (1992).

${ }^{14}$ K. A. Brakke, Surface Evolver Manual, Version 2.70 (2013), available at http://www.susqu.edu/brakke/evolver/downloads/evolver2.70-Win32.zip.

${ }^{15}$ S. Wu, J. Phys. Chem. 74, 632 (1970).

${ }^{16}$ See supplementary material at http://dx.doi.org/10.1116/1.4896480 for geometry file, simulation file, and simulation video. 